\title{
Effects of High Energy Milling on the Carbon- coated Lithium Iron Phosphate Precursor Nature
}

\author{
Peng Chen ${ }^{1, \mathrm{a}}$, Rong Pan ${ }^{2, \mathrm{~b}}$, Yazhen Ren ${ }^{2, \mathrm{c}}$, Zhigang $\mathrm{Wu}^{2, \mathrm{~d}}$, Min Zhang ${ }^{3, \mathrm{e}}$, Kejie Zhang ${ }^{*}, \mathrm{f}$ \\ ${ }^{1,2,3}$ Nanjing Institute of Technology, Nanjing, Jiangsu Province, China \\ a1964472154@qq.com, ${ }^{\mathrm{b}} 154371995 @$ @q.com, ${ }^{\mathrm{c}} 1607516047 @$ qq.com, ${ }^{\mathrm{d}} 429448142 @$ @q.com, \\ e1430346079@qq.com, f346082291@qq.com
}

\begin{abstract}
LiFePO}_{4}$ precursor is produced in planetary milling with $\mathrm{LiOH} \cdot \mathrm{H}_{2} \mathrm{O}, \mathrm{H}_{3} \mathrm{PO}_{4}, \mathrm{Fe}_{2} \mathrm{O}_{3}$ and sucrose, which is characterized by X-ray diffraction, scanning electron microscope(SEM), laser particle size analyzer (LPSA). The results showed that the particle size was submicron level, the LPSA data showed that there was double peak of the sample, which the big one appeared around $0.66 \mu \mathrm{m}$ and the small one appeared around $1.71 \mu \mathrm{m}$ and the positions did not change with the change of parameters. The mixture was conductive to obtain narrow-size-range $\mathrm{LiFePO}_{4}$.
\end{abstract}

Keywords: Precursor of $\mathrm{LiFePO}_{4}$, Planetary ball milling, $\mathrm{LiFePO}_{4}$, Particle size distribution.

\section{Introduction}

Olivine lithium iron phosphate $\left(\mathrm{LiFePO}_{4}, \mathrm{LFP}\right)$ is used in lithium-ion batteries as cathode material $^{[1,2]}$. LFP holds the desirable merits of abundant raw materials, non-toxicity, high thermal stability, suitable voltage of $3.4 \mathrm{~V}\left(\mathrm{vs} \mathrm{Li}^{+} / \mathrm{Li}\right)$ and theoretical capacity of 170 $\mathrm{mA} \cdot \mathrm{h} / \mathrm{g}^{[3,4]}$. It meets both demands of high energy density and environmental friendliness and is an adequate cathode for power battery and stationary storage of electrical energy generated by renewable power ${ }^{[5,6,7]}$. However, the electronic conductivity of LFP ranges from $10^{-9}$ to $10^{-10} \mathrm{~s} / \mathrm{cm}$ and the lithium-ion diffusion rate at $1.8 \times 10^{-14} \mathrm{~cm}^{2} / \mathrm{s}$ which is low and determine its poor electrochemical performance under high rates ${ }^{[8,9]}$. Presently, these disadvantages are overcome by carbon coating, metal-ions doping and nano structure ${ }^{[10,11,12]}$.

Generally, mechanical activation was realized by high-energy ball milling. The milling worked by using mechanical force to refine the particle size, increase the contact area and makes the lattice occur defects. At the same time, it fully mixed materials so that it promoted the carbon-thermal reduction reaction. The precursor, produced by mixing lithium source, phosphorus source, iron source and carbon source, was heated in inert atmosphere and finally became carbon-coated LFP cathode material ${ }^{[13]}$. The performance of the sample will be better with the morphology becoming more uniform and the particle size decreasing ${ }^{[14,15,16]}$. But so far there has been few researches reported the milling process

*Corresponding author: 346082291@qq.com 
effect on the precursor. The milling effects on the crystal form, morphology, particle size and distribution of LFP are analysised in the paper.

\section{Experiment}

\subsection{Preparation of materials.}

Ceramic balls of different dimension were used, including the big ball of $9 \mathrm{~mm}$ diameter, the medium ball of $6 \mathrm{~mm}$ diameter, and the small ball of $5 \mathrm{~mm}$ diameter. The number ratio of the small-milling-ball, medium-milling-ball and big-milling-ball was 1: 1: 1. Volume of the stainless steel milling tank is $75 \mathrm{~cm}^{3}$. The milling medium was ethanol. $\mathrm{LiOH} \cdot \mathrm{H}_{2} \mathrm{O}$, $\mathrm{H}_{3} \mathrm{PO}_{4}, \mathrm{Fe}_{2} \mathrm{O}_{3}$, sucrose and ethanol were purchased from Chengdu Kelong Chemical Reagent Factory. All reagents were of analytical grade and used as received without further purification.

\subsection{Experimental Procedure.}

Certain amount of $\mathrm{LiOH} \cdot \mathrm{H} 2 \mathrm{O}$, $\mathrm{H} 3 \mathrm{PO} 4$ and $\mathrm{Fe} 2 \mathrm{O} 3$, which were mixed in an agate mortar with ethanol, were weighed according to molar ratio of 1.1: $1: 0.5$. The mixture was heated in $100{ }^{\circ} \mathrm{C}$ vacuum oven. The precursor was obtained by milling the mixture and some sucrose. The milling parameters were preliminarily determined as followed: ball-topowder weight ratio of 6: 1, milling for 3 hours (milled for $20 \mathrm{~min}$, paused for $10 \mathrm{~min}$, milling and reversing, 9 cycles), $350 \mathrm{r} / \mathrm{min}$. One of the parameters for the test was changed, while the other parameters unchanged.

\subsection{Experimental device and testing equipment.}

A single tank planetary high energy ball mill (Pulverisette-6, FRITSCH, Germany) was used to mill samples. A laser particle size analyzer (BT9300H, Dandong Better Instrument Co., China) was used to test the samples' particle size and distribution. The test range was from $0.1 \mu \mathrm{m}$ to $100 \mu \mathrm{m}$. A cold field emission scanning electron microscopy (JSM-6360LV, JEOL, Japan) was used to observe morphology of samples. An X-ray diffraction (UltimaIV, Rigaku, Japan) was used to analyze crystal structure with $\mathrm{K} \alpha$ and wavelength for $0.5146 \mathrm{~nm}$.

\section{Results and discussion}

Fig. 1 presented XRD patterns of the precursor and carbon-coated LFP produced by roasting precursor at $700{ }^{\circ} \mathrm{C}$ for 9 hours in nitrogen atmosphere. Fig. 1(a) showed that there were sharp diffraction peaks at $2 \theta=24.14^{\circ}, 33.17^{\circ}, 35.59^{\circ}, 40.83^{\circ}, 49.41^{\circ}, 54.09^{\circ}, 62.48^{\circ}$, $64.03^{\circ}$, which indicated that crystallization degree of $\mathrm{Fe}_{2} \mathrm{O}_{3}$ (JCPDS card number 87-1164) was deep. Materials had not changed, because milling only mixed materials, grinding and other physical changes, without chemical change. The crystal growth did not occur in $\mathrm{H}_{3} \mathrm{PO}_{4}$ and $\mathrm{LiOH}$, so there were not diffraction peaks in the XRD patterns. Fig. 1(b) showed that there were sharp diffraction peaks at $2 \theta=17.09^{\circ}, 20.80^{\circ}, 22.61^{\circ}, 25.58^{\circ}, 29.73^{\circ}$, $32.27^{\circ}, 35.59^{\circ}, 36.59^{\circ}, 42.19^{\circ}, 52.56^{\circ}, 61.86^{\circ}$, which meant that crystallization degree of LFP (JCPDS card number 40-1499) is high. Diffraction peak obtained by test is the same as the one that was listed in the X-ray powder diffraction data file, which showed that olivinetype LFP was obtained and the crystallinity was excellent. Diffraction peak of carbon was not shown, which pointed out that residual carbon was amorphous phase. 


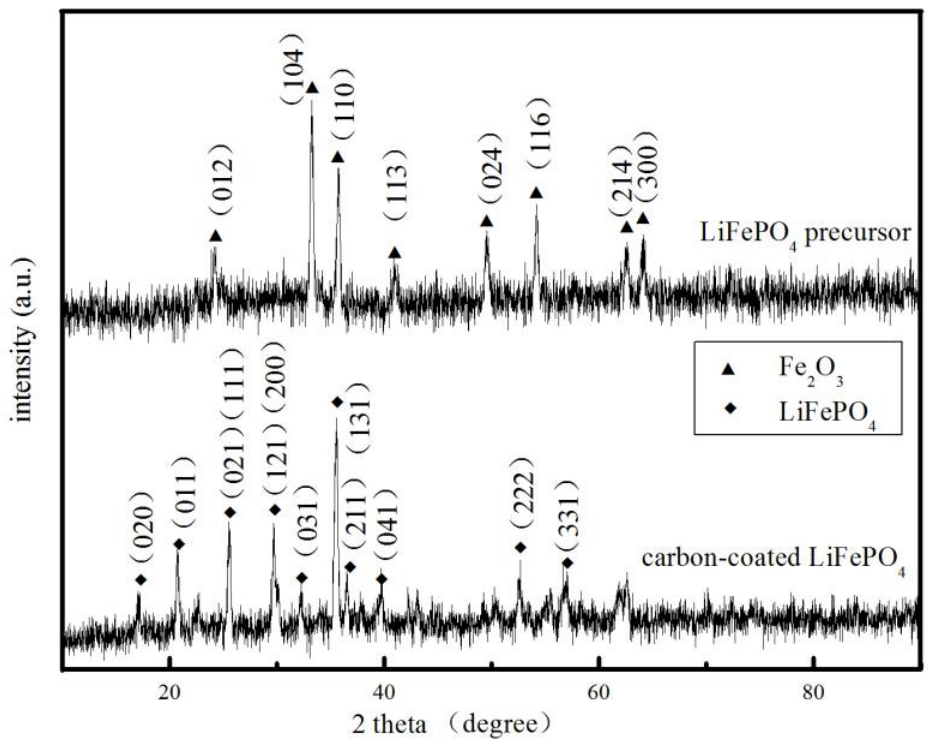

Fig. 1 XRD patterns of the precursor and carbon-coated LFP $\left(700{ }^{\circ} \mathrm{C}, 9 \mathrm{~h}\right)$

Fig. 2 showed SEM patterns of the precursor and LFP. From Fig. 2, after milling, precursor micro-morphology is uniform, submicron and spherical particle and slight agglomeration existed. The particle size of LFP was close to the size of precursor, which indicated that the carbon limited growth of LFP crystal and made morphology of LFP uniform.

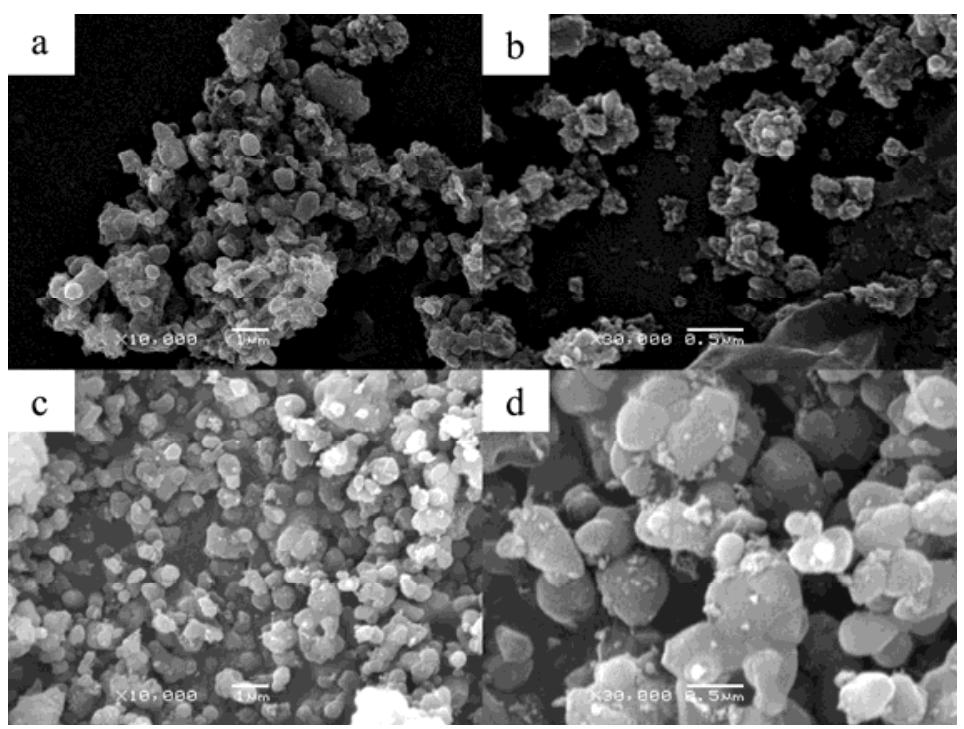

Fig. 2 SEM patterns of the precursor and LFP a,b. the precursor, c,d. LFP

Fig. 3 is a group of patterns of the particle size of the precursor. The three figures demonstrated that the particle size distribution presented something interesting with varying parameters: the particle size mainly concentrated in $0 \sim 4 \mu \mathrm{m}$. When particle size smaller 
than $2.5 \mu \mathrm{m}$, the double peak occurred of which the big one appeared around $0.66 \mu \mathrm{m}$ and the small one appeared around $1.71 \mu \mathrm{m}$ and positions did not change with the change of parameters, which indicated that the particle size distribution was in relative steady state and particle fragmentation and agglomeration was competitive. The result of the competition was that particle size concentrated to $0.66 \mu \mathrm{m}$ and $1.70 \mu \mathrm{m}$. When the particle size was bigger than $4 \mu \mathrm{m}$, the distribution curve became flat and low.
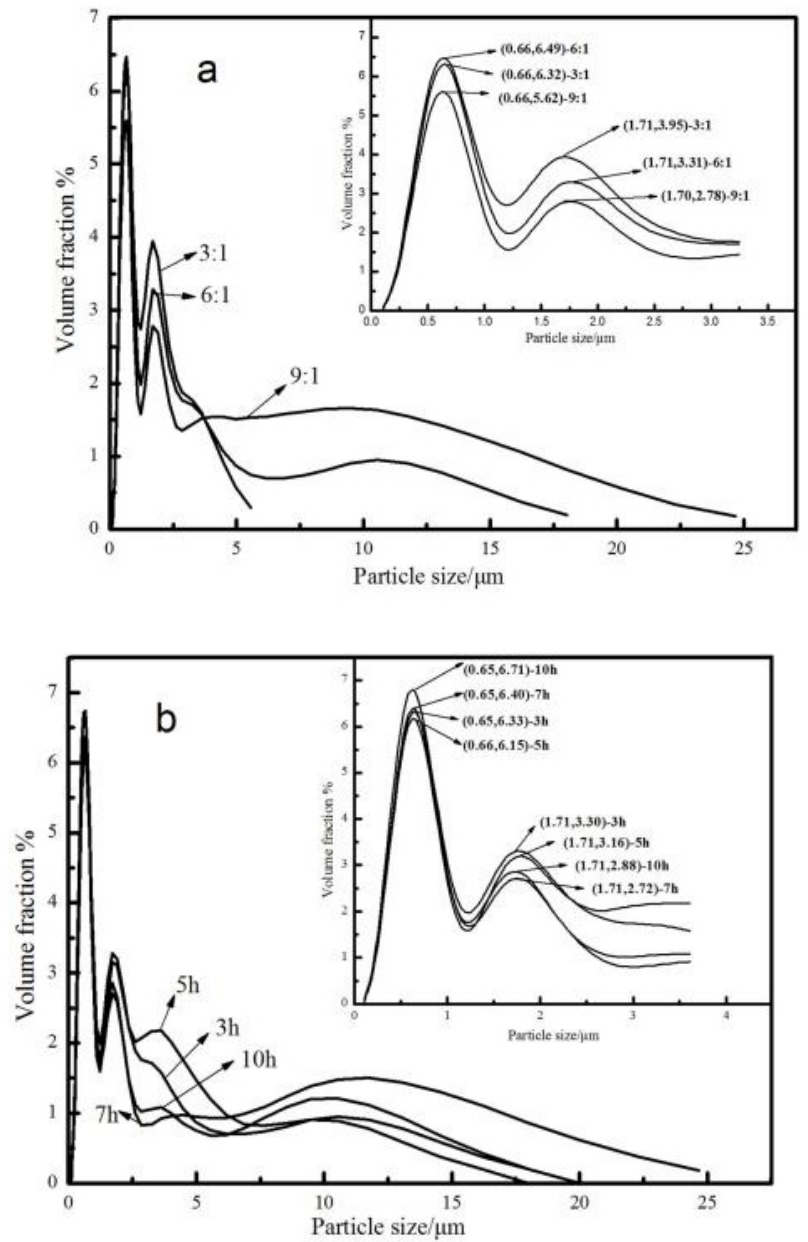


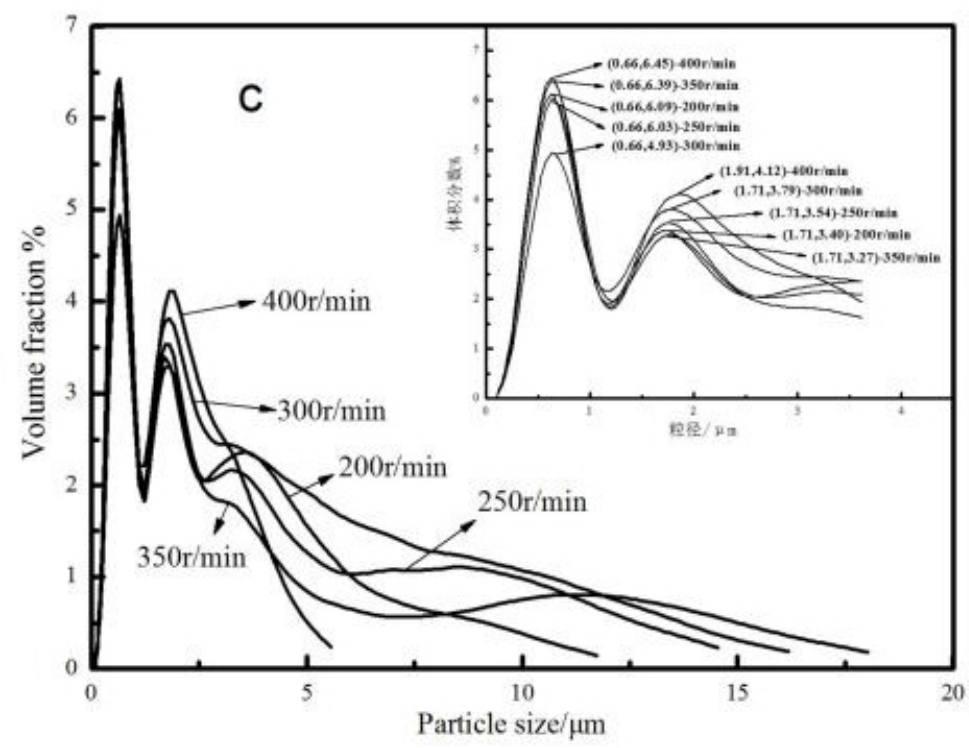

Fig. 3 patterns of the particle size of the precursor produced in the different parameters a. changing ball-to-powder weight ratio, b. changing milling time, c. changing speed

\section{Conclusion}

The particle size of the precursor was submicron by planetary ball milling which can make the carbon source uniformly mixed with the precursor. So that, the product can completely react during heated. The morphology of precursor was spherical after milling, and olivine LFP could be produced by heating the precursor.

There were following characteristics of particle size distribution: the size of material was in the range of $0 \sim 4 \mu \mathrm{m}$. The distributed curve became flat and low when the size was bigger than $4 \mu \mathrm{m}$. The particle breakage and agglomeration competed for the result of particle gathered in the size of $0.66 \mu \mathrm{m}$ and $1.70 \mu \mathrm{m}$, so the particle size distribution was in a relatively stable state.

\section{Acknowledgment}

This work was supported by the major projects of Nanjing Institute of Technology (CKJA201303), the General Program of Jiangsu Provincial Department of Education (15KJB430015), the Students Innovative Projects of Nanjing Institute of Technology(N20150229), the Students Innovative Projects in Jiangsu Province(201511276066X).

\section{References}

1. K. Padhi, K. S. Nanjundaswamy, J. B. D. Goodenough, Phospho-olivines as positive - electrode materials for rechargeable lithium batteries, J. Electrochem. Soc. 144 (1997) 1188-1194.

2. D. Jiang, X. Zhang, S. Lu, Research on process of preparation and performance of iron phosphate as precusor of lithium iron phosphate, Rare Metals. 30 (2011) 52-54. 
3. X. Liao, J. Yu, L. Gao, Electrochemical study on lithium iron phosphate/hard carbon lithium-ion batteries, J. Solid State Electr. 16 (2012) 423-428.

4. Mauger, C. Julien, Surface modifications of electrode materials for lithium-ion batteries: status and trends, Ionics. 20 (2014) 751-787.

5. J. M. Patete, M. E. Scofield, V. Volkov, et al., Ambient synthesis, characterization, and electrochemical activity of $\mathrm{LiFePO}_{4}$ nanomaterials derived from Iron phosphate intermediates, Nano Res. 8 (2015) 2573-2594.

6. M. J. Armstrong, O'D. Colm, W. J. Macklin, Evaluating the performance of nanostructured materials as lithium-ion battery electrodes, Nano Res. 7 (2014) 1-62.

7. L. Noerochim, A. O. Yurwendra, D. Susanti, Effect of carbon coating on the electrochemical performance of $\mathrm{LiFePO}_{4} / \mathrm{C}$ as cathode materials for aqueous electrolyte lithium-ion battery, Ionics. 17 (2015) 1-6.

8. G. Sun, B. Jin, G. Sun, et al., Characteristics of lithium iron phosphate mixed with nano-sized acetylene black for rechargeable lithium-ion batteries, J. Appl. Electrochem. 41 (2011) 99-106.

9. Y. Chang, C. Peng, I. Hung, Effects of particle size and carbon coating on electrochemical properties of $\mathrm{LiFePO}_{4} / \mathrm{C}$ prepared by hydrothermal method, J. Mate. Sci. 49 (2014) 6907-6916.

10. Zhao, X. Yu, R. Cai, et al., Solution combustion synthesis of high-rate performance carbon-coated lithium iron phosphate from inexpensive iron (III) raw material, J. Mate. Chem. 22 (2012) 2900-2907.

11. T. F. Yi, X. Y. Li, H. Liu, et al., Recent developments in the doping and surface modification of $\mathrm{LiFePO}_{4}$ as cathode material for power lithium ion battery, Ionics. 18 (2012) 529-539.

12. J, Hassoun, F, Bonaccorso, M. Agostini, et al., An advanced lithium-ion battery based on a graphene anode and a lithium iron phosphate cathode, Nano Lett. 14 (2014) 49014906.

13. L. Damen, F. De Giorgio, S. Monaco, et al., Synthesis and characterization of carboncoated $\mathrm{LiMnPO}_{4}$ and $\mathrm{LiMn}_{1-} \mathrm{Fe}_{\mathrm{x}} \mathrm{PO}_{4}(\mathrm{x}=0.2,0.3)$ materials for lithium-ion batteries, $\mathrm{J}$. Power Sources. 218 (2012) 250-253.

14. M. S. Yoon, M. Islam, Y. M. Park, et al., Effect of synthesizing method on the properties of $\mathrm{LiFePO}_{4} / \mathrm{C}$ composite for rechargeable lithium-ion batteries, Electron. Mater. Lett. 9 (2013) 187-193.

15. Z. Fu, F. Yu, S. Ling, Influence of Wet Milling Process Parameters on the Zirconium Silicate Average Particle Size, Ti. Ind. Prog.31 (2014) 41-44.

16. P. Liu, A. Wang, L. Yan, Effect of milling time on morphology of composite powders as well as microstructure and properties of large size ratio SiC/6061 Al composites, Mater. Sci. Eng. Powder Metal. 19 (2014) 523-529. 